\title{
The Semiempirical Method for Finding Thermal Characteristics of Simple Crystals
}

\author{
P. SCHAROCH* \\ Institute of Physics, Wrocław University of Technology \\ Wyb. Wyspiańskiego 27, 50-370 Wrocław, Poland
}

(Received July 12, 2004)

\begin{abstract}
A method for ab initio (using density functional theory) study of thermal properties of crystalline solids, based on the quasiharmonic approximation, is briefly summarized. On that basis the semiempirical method is proposed which combines the $a b$ initio calculation of the static total energy with the Einstein model of crystal vibration. The Murnaghan equation of states is used as an analytical model for the static total energy. An exponential form of the phonon energy versus volume dependence is introduced which was proved to perform very well. Two parameters appearing in the model are found by fitting to easily available experimental data (tabular or measured). The method then provides thermodynamic characteristics in a large range of temperatures and pressures. On the other hand, the corrections due to the zero-point vibration are provided to some first principles results, like lattice parameters or bulk modulus. An interesting outcome of the model is the pressure dependence of the overheating temperature, for relatively low pressures. Tests performed on the example of fcc aluminum show remarkably good agreement of the results with experimental data. Therefore the method offers a handy tool for fast analysis of thermodynamics of simple crystalline systems, omitting the first principles evaluation of the phonon energies.
\end{abstract}

PACS numbers: 65.40.-b, 64.10.+h, 31.15.Ar

\section{Introduction}

The computational methods based on the density functional theory (DFT) (see e.g. [1]), developing quickly over the last decades, provide very promising opportunities to study various aspects of polyatomic systems physics from first

*e-mail: scharoch@rainbow.if.pwr.wroc.pl 
principles, i.e. using only fundamental theories and universal constants. At the same time the semiempirical methods, which combine the $a b$ initio calculations with empirical models, develop, offering an alternative research tool in the cases where the pure $a b$ initio calculations require a big computational effort. This paper deals with a particular topic: thermal properties of crystals. The approach applied is known in the literature as the quasiharmonic approximation. Several papers have appeared so far in which the ab initio calculations of crystal thermodynamics have been reported (e.g. [2-6]). The computational procedure involves the evaluation of the phonon energies, which is a quite demanding computational task (e.g. [7-10]). In this paper a significantly simplified model is proposed which omits the evaluation of phonons dispersion but includes the crucial, as it turns out, first principles dependence of static total energy vs. volume which can be calculated fairly easily. The lattice dynamics is represented by the Einstein model in which phonon energy is related to crystal volume via exponential function. The model contains two parameters: the phonon energy at static equilibrium of a crystal and the factor in the exponential function modeling the volume dependence of the phonon energy. They can be fitted using easily available tabular data (or data obtained from simple measurements). The method also provides corrections due to the zero-point vibration to some ab initio results. As an additional outcome of the model, the pressure dependence of the overheating temperature (the temperature at which a crystal loses its mechanical stability), for relatively low pressures, is obtained. The model has been tested on the example of aluminum and provides very good overall thermodynamic characteristics. Thus the method may serve as a handy tool for fast analysis of the thermodynamics of materials when the knowledge of their thermal properties in the large range of temperatures and pressures are needed. In particular, it is very useful when studying crystal surfaces or thin layers where the characteristics of the bulk-substrate is needed.

\section{Thermodynamics}

The thermodynamics analysis is performed within a concept of canonical ensemble. The following notation is introduced: $X_{i}=\exp \left(-\beta E_{i}\right)$, where the phonon energy $E_{i}=\hbar \omega_{i}$ and $\omega_{i}$ is the frequency of the normal mode of index $i, \beta=1 / k_{\mathrm{B}} T, T$ is the temperature of the system and $k_{\mathrm{B}}$ - the Boltzmann constant. The single mode quantities: partition function $Z_{i}$, free energy $F_{i}$, internal energy $U_{i}$, entropy $S_{i}$, and specific heat $C_{i}$ assume the known forms: $Z_{i}=$ $\sqrt{X_{i}} /\left(1-X_{i}\right), \quad F_{i}=-\ln \left(Z_{i}\right) / \beta, \quad U_{i}=E_{i}\left(1+X_{i}\right) /\left[\left(1-X_{i}\right) 2\right], \quad S_{i}=\left(U_{i}-\right.$ $\left.F_{i}\right) / T, C_{i}=k_{\mathrm{B}} \beta^{2} E_{i}{ }^{2} X_{i} /\left(1-X_{i}\right)^{2}$, and the respective quantities for the whole system are: $Z=\prod_{i} Z_{i}, F=\sum_{i} F_{i}+E_{0}^{\text {tot }}, U=\sum_{i} U_{i}+E_{0}^{\text {tot }}, S=\sum_{i} S_{i}, C_{V}=$ $\sum_{i} C_{i}$, where $E_{0}^{\text {tot }}$ is the total energy of the system at static equilibrium and the summation (product) runs over all normal modes.

The nonlinear effects, like thermal expansion, are accounted for indirectly by assuming that phonon frequencies depend on volume, $\omega_{i}=\omega_{i}(V)$. It is a com- 
mon practice to introduce a dimensionless quantity called the Grüneisen number, defined as $\gamma_{i}=-\mathrm{d}\left[\ln \left(\omega_{i}\right)\right] / \mathrm{d}[\ln (V)]$. The Grüneisen number is normal mode specific, therefore must be indexed by $i$. The expressions for various thermodynamic quantities can be derived now from standard definitions, e.g.:

- The equation of state takes the form (the Mie-Grüneisen equation of state)

$$
P(V, T)=P_{0}(V)+\frac{1}{V} \sum_{i} U_{i} \gamma_{i},
$$

where $P_{0}$ is the static contribution to pressure, equal to $-\partial E^{\text {tot }}(V) / \partial V$. In this formula and all the formulae below the summation runs over all normal modes.

- The isothermal modulus of elasticity

$$
\begin{aligned}
B_{T} & =-V\left(\frac{\partial P}{\partial V}\right)_{T}=B_{T 0}+\left(P-P_{0}\right)+\frac{1}{V} \sum_{i} \gamma_{i}^{2}\left(U_{i}-C_{i} T\right) \\
& -\sum_{i} U_{i} \frac{\partial \gamma_{i}}{\partial V}
\end{aligned}
$$

where $B_{T 0}$ is a respective static contribution.

- The isobaric (at constant pressure) volume thermal expansion coefficient

$$
A_{P}=\frac{1}{V}\left(\frac{\partial V}{\partial T}\right)_{P}=\frac{1}{V B_{T}} \sum_{i} \gamma_{i} C_{i} .
$$

In the quantities above the static total energy as a function of volume, $E^{\text {tot }}(V)$ (together with its first and the second derivatives), appears. In principle, it can be scanned directly in $a b$ initio calculations. Here the analytic form for this function, resulting from the Murnaghan equation of state [11], has been used to fit $a b$ initio data

$$
E^{\mathrm{tot}}(V)-E^{\mathrm{tot}}\left(V_{0}\right)=\frac{B_{0} V}{B_{0}^{\prime}}\left[\frac{\left(V_{0} / V\right)^{B_{0}^{\prime}}}{B_{0}^{\prime}-1}+1\right]-\frac{B_{0} V_{0}}{B_{0}^{\prime}-1},
$$

where $V_{0}, B_{0}, B_{0}^{\prime}$ are the parameters corresponding to the static equilibrium geometry and they denote respectively: volume, modulus of elasticity, and its derivative with respect to pressure. With this equation the static contribution to pressure takes the form: $P_{0}(V)=\left(B_{0} / B_{0}^{\prime}\right)\left[\left(V_{0} / V\right)^{B_{0}^{\prime}}-1\right]$, and the static contribution to bulk modulus $\left(B_{T 0}\right)$ is: $B_{T 0}(V)=B_{0}\left(V_{0} / V\right)^{B_{0}^{\prime}}$.

\section{Phonon energy vs. volume dependence}

Although in principle the phonon energy vs. volume dependence can be scanned $a b$ initio directly, usually a model function is used like power law or the Taylor series either linear or up to second-order term (e.g. [2]). Here the 
exponential function is proposed for that purpose and according to the experience of the author, including $a b$ initio calculations in large range of volumes, such model describes very well the dependence of phonon frequency, $\omega_{i}$, on the relative volume deviation, $x=\left(V-V_{0}\right) / V_{0}$. It is then given by: $\omega_{i}(x)=\omega_{i}(0) \exp \left(-\alpha_{i} x\right)$. The parameters $\omega_{i}(0)$ and $\alpha_{i}$ are normal mode specific and can be found from ab initio calculations.

With such a choice of the model function the Mie-Grüneisen equation of state and the bulk modulus formula simplify significantly

$$
\begin{aligned}
& P(V, T)=P_{0}(V)+\frac{1}{V_{0}} \sum_{i} U_{i} \alpha_{i}, \\
& B_{T}=B_{T 0}+\frac{V}{V_{0}^{2}} \sum_{i} \alpha_{i}^{2}\left(U_{i}-C_{i} T\right) .
\end{aligned}
$$

\section{Loss of mechanical stability (overheating)}

An interesting outcome of the model described above is the phonon modes softening limit, which can be associated with the loss of mechanical stability of a crystal (overheating). One can presume that the loss of mechanical stability can be caused by softening of at least one normal mode. A mode softening means that its energy tends to zero. It is easy to find the asymptotic behavior in that limit of functions introduced previously, e.g.: $X_{i} \rightarrow 1, U_{i} \rightarrow k_{\mathrm{B}} T, C_{i} \rightarrow k_{\mathrm{B}}, P \rightarrow$ $-B_{0} / B_{0}^{\prime}, B_{T} \rightarrow 0$. The equation of state assumes a particular simple form

$$
P=-B_{0} / B_{0}^{\prime}+D T_{\mathrm{cr}},
$$

where $D=k_{\mathrm{B}} \sum_{i} \alpha_{i} / V_{0}$ is a constant and $T_{\text {cr }}$ is a critical (overheating) temperature. Thus, the equation above shows a linear dependence of the critical temperature vs. pressure and gives an estimation of the value of pressure which begins to influence the critical temperature (it must be of the order of $B_{0} / B_{0}^{\prime}$ ). The value of the constant $D$, although it is given by the explicit formula, can be obtained from the equation above when it is rewritten for the critical temperature at zero pressure $\left(T_{\mathrm{cr}}^{0}\right): D=B_{0} / B_{0}^{\prime} T_{\mathrm{cr}}^{0}$. Therefore, the final formula for the critical temperature vs. pressure takes the form

$$
T_{\mathrm{cr}}=T_{\mathrm{cr}}^{0}\left(\frac{B_{0}^{\prime}}{B_{0}} P+1\right) \text {. }
$$

The values $B_{0}$ and $B_{0}^{\prime}$ can be easily calculated from first principles, whereas for $T_{\mathrm{cr}}^{0}$ an easily available experimental melting temperature can be used. As will be shown below, the model works very well for fcc aluminum at relatively low pressures.

\section{Method of computation}

The calculations must begin with finding the ab initio static total energy, $E^{\text {tot }}(V)$, as a function of system volume. This can be done by one of the available 
$a b$ initio computer packages (e.g. [12, 8, 13]). In the next step, when performing pure $a b$ initio calculations, one has to calculate from first principles the phonon frequencies at certain set of $k$-points in the first Brillouin zone (BZ). The summation in Eqs. (1)-(3) can be performed with the use of the Monkhorst-Pack [14] scheme. In this paper, however, only the single mode (triply degenerate) properties of the model are analyzed. This corresponds, on the one hand, to the choice of only one special $k$-point in the Monkhorst-Pack summation, and on the other hand it is equivalent to the application of the Einstein model of crystal vibration.

\section{The single mode properties of the model}

In this section some examples of single-mode calculations, illustrating the properties of the model, are presented.

The set of parameters appearing in Eq. (4) has been found by fitting the function to the numerical data of ab initio total energy of aluminum crystal. The $a b$ initio calculations have been performed with the use of fhi98md, fhiPP packages $[12,15]$, and with the exchange-correlation functional given by GGA-PBE [16]. The following values of the parameters have been found: $V_{0}=16.285 \AA^{3}, B_{0}=$ $76.1 \mathrm{GPa}, B_{0}^{\prime}=5.6$.

As an example the entropy and the volume thermal expansion coefficient, respectively, calculated as functions of temperature and at zero pressure are shown in Figs. 1, 2. The graphs denoted by (a) show results obtained at constant frequency $\omega=6 \mathrm{THz}$ and at 3 different parameters $\alpha=1,2,3$. The graphs denoted by (b) show results obtained at constant parameter $\alpha=2$, and at 3 different frequencies $\omega=1,6,11 \mathrm{THz}$. The chosen values are typical of polyatomic systems. The results presented show the sensitivity of the model on the variation of the parameters $\omega$ and $\alpha$. The observed properties have been used in the described
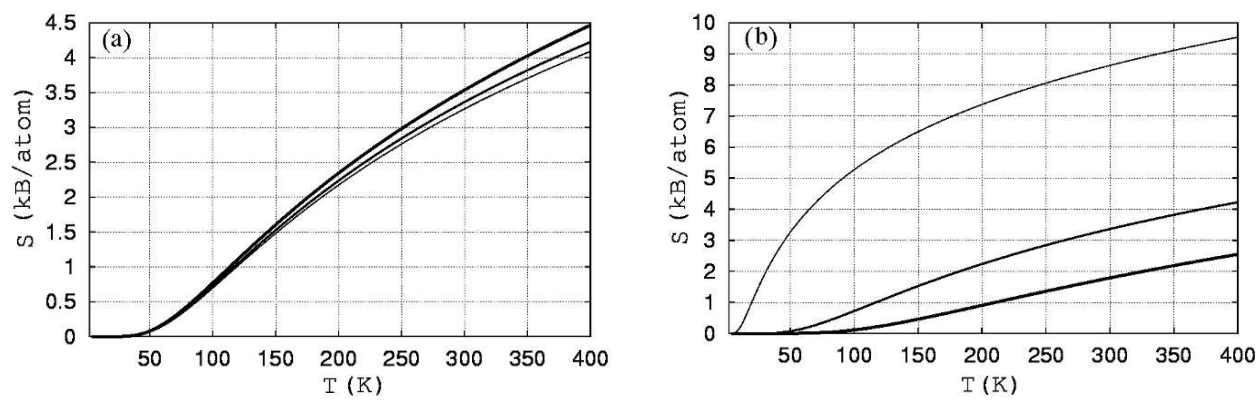

Fig. 1. The entropy. (a) $\omega=6 \mathrm{THz}$ and $\alpha=1,2,3$, increasing with increasing line thickness; (b) $\alpha=2$ and $\omega=1,6,11 \mathrm{THz}$, increasing with increasing line thickness. As might be expected, the frequency has a significant influence on entropy (b). The entropy depends weakly on $\alpha$ parameter (a). 

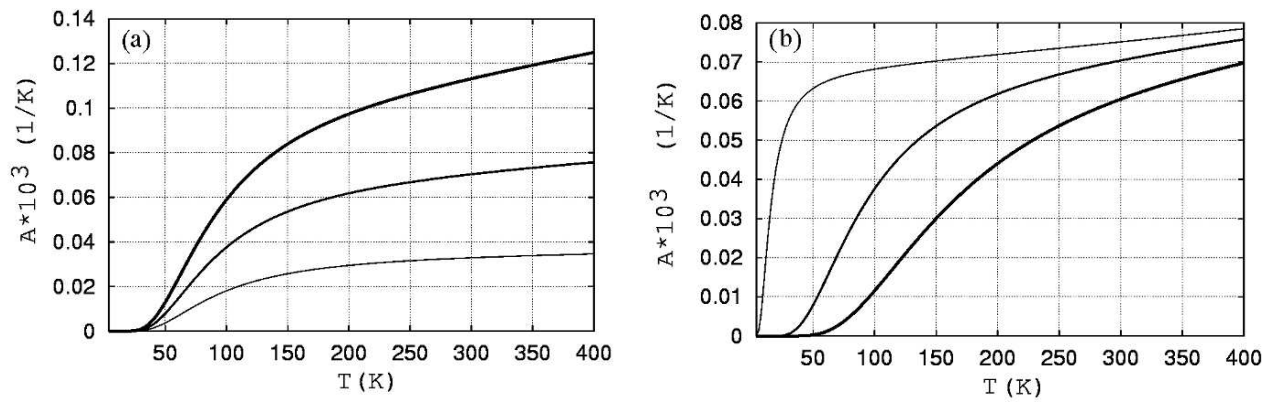

Fig. 2. The volume thermal expansion coefficient (Eq. (3)). (a) $\omega=6 \mathrm{THz}$ and $\alpha=1,2,3$, increasing with increasing line thickness; (b) $\alpha=2$ and $\omega=1,6,11 \mathrm{THz}$, increasing with increasing line thickness. Both: frequency (b) and $\alpha$ parameter (a), strongly influence the thermal expansion.

below semiempirical method. In particular, the weak dependence of entropy on $\alpha$ makes the fitting of the $\omega$ parameter very easy.

\section{The semiempirical method}

The presented above features of the model can be used to fit the parameters $\alpha$ and $\omega(0)$ to experimental data for entropy and thermal expansion coefficient. The method has been applied to Al fcc crystal. The ab initio total energy vs. volume has been calculated as described previously. The following values of the parameters have been found by fitting to tabular data: $\omega=5.874 \mathrm{THz}$ (by fitting to experimental entropy at $298 \mathrm{~K}: S=28.3 \mathrm{~J} /(\mathrm{mol} \mathrm{K}))$ and $\alpha=1.998$ (by fitting to the thermal expansion coefficient at $291 \mathrm{~K}$ and zero pressure: $A=$ $\left.23.32 \times 10^{-6} 1 / \mathrm{K}\right)$.
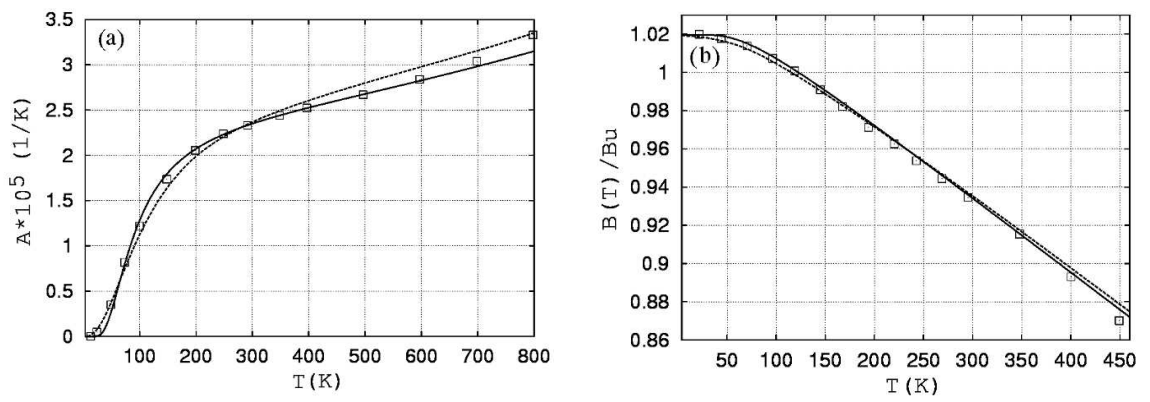

Fig. 3. The linear thermal expansion coefficient (a) and isothermal bulk modulus (normalized to the value of static contribution at zero temperature volume, $B u=66.16 \mathrm{GPa})(\mathrm{b})$, vs. temperature, in Al; solid line: the graph obtained by the method described in this paper; broken line: the results of pure ab initio calculations [2]; points: experimental data [17, 18]. 

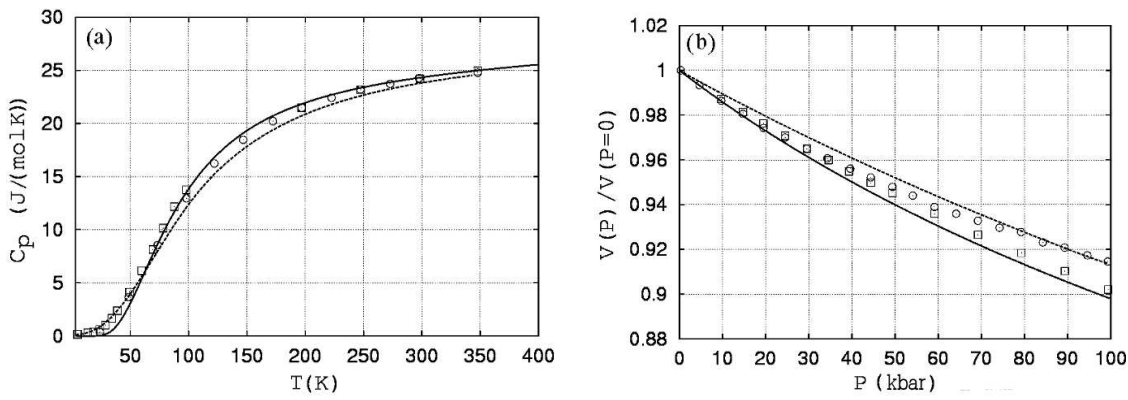

Fig. 4. Temperature dependence of isobaric specific heat (a) and pressure dependence of relative change in volume (b), in $\mathrm{Al}$; solid line: the curve obtained by the method described in this paper; broken line: the results of pure ab initio calculations [2]; points: experimental data $[18,19,17]$.

Some examples of the results are presented and compared with the pure $a b$ initio calculations [2] and experimental data: in Figs. 3, 4. The method provides very good thermal characteristics of aluminum in a large range of temperatures and pressures. Some corrections to the ab initio results due to the zero-point vibration are also provided. For example, the static equilibrium value of the bulk modulus is equal to $76.1 \mathrm{GPa}$. However, at zero temperature, due to the zero-point vibration, the static contribution is significantly decreased: $72.2 \mathrm{GPa}$ and the resultant value of the bulk modulus is $73.6 \mathrm{GPa}$ in which the purely vibrational contribution is on the order of $2 \%$. The zero-point vibration correction to the $a b$ initio lattice constant equals $0.32 \%$ and leads to the value of the lattice constant: $4.036 \AA$, differing from the experimental one $(4.049 \AA)$ only by $0.3 \%$.

As the last example the critical temperature vs. pressure has been calculated. The experimental melting temperature at zero pressure $(933.47 \mathrm{~K})$ has been used to fit $\alpha$ parameter. As Fig. 5a shows, the model gives correct linear dependence
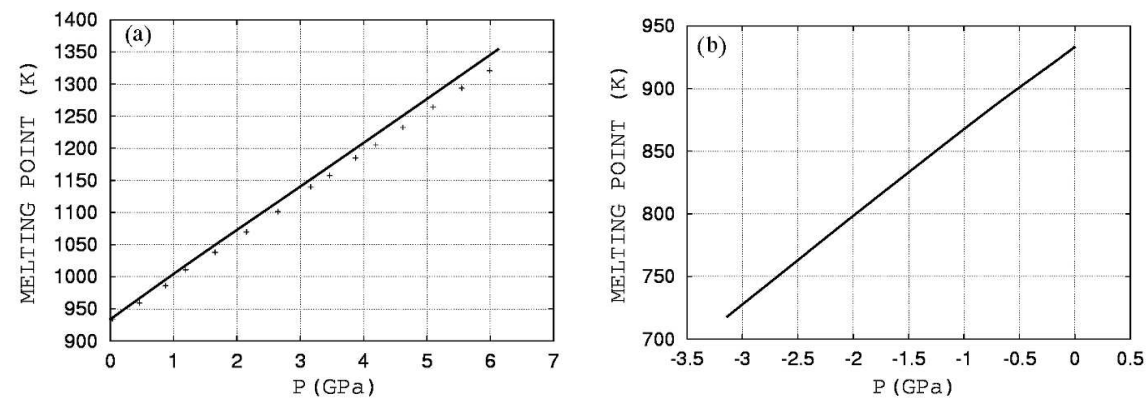

Fig. 5. Melting temperature vs. pressure for positive (a) and negative (b) pressures, in $\mathrm{Al}$; solid line: the graph obtained with the method described in this paper; points: data according to [20]. 
of melting temperature vs. pressure at the initial part of the melting curve. This fact seems to confirm the conclusions from previous discussion. However, one must admit that at higher pressures a large deviation from linearity is observed [20], which is not exhibited by the model. Finally, Fig. 5b also shows the critical curve corresponding to negative pressure. A significant linear decrease in melting temperature can be seen which points at the fact that a local negative strain in material may result in the local decrease in the melting temperature.

\section{Conclusions}

In the paper the model for $a b$ initio analysis of thermal properties of crystals is described. The proposed semiempirical method provides very good estimations of thermal characteristics and appears to be a handy tool e.g. when the characteristics are needed for some other purposes like studying the crystal surface. The advantage of this approach is that it is fast and relatively simple. The method, however, should not be thought of as a universal one, it has been shown to work well for a simple fcc crystal but its universality remains an open question and will be a subject of further tests. The semiempirical method based on the Debye model is a straightforward extension of the method presented here and will be a subject of further study.

\section{Acknowledgments}

The author would like to thank Adam Kiejna [21] for his comments concerning the manuscript and Artem Oganov [22] for his very helpful remarks and critics. The numerical calculations for this work were done in Wrocław Networking and Computing Centre (WCSS).

\section{References}

[1] M.C. Payne, M.P. Teter, D.C. Allan, T.A. Arias, J.D. Joannopoulos, Rev. Mod. Phys. 64, 1045 (1992).

[2] A. Debernardi, M. Alouani, H. Dreysse, Phys. Rev. B 63, 064305-1 (2001).

[3] J. Xie, S. de Gironcoli, S. Baroni, M. Scheffler, Phys. Rev. B 59, 965 (1999).

[4] A.A. Quong, A.Y. Liu, Phys. Rev. B 56, 7767 (1997).

[5] S. Biernacki, M. Scheffler, Phys. Rev. Lett. 63, 290 (1989).

[6] A.R. Oganov, P.I. Dorogokupets, Phys. Rev. B 67, 224110 (2003).

[7] G.J. Ackland, M.C. Warren, S.J. Clark, J. Phys, Condens. Matter 9, 7861 (1997).

[8] S. Baroni, S. de Gironcoli, A. Dal Corso, Rev. Mod. Phys. 73, 515 (2001).

[9] K. Parlinski, http://wolf.ifj.edu.pl/phonon/.

[10] P. Scharoch, K. Parlinski, A. Kiejna, Acta Phys. Pol. A 97, 349 (2000). 
[11] F.D. Murnaghan, Proc. Natl. Acad. Sci. USA 30, 244 (1944).

[12] M. Bockstedte, A. Kley, J. Neugebauer, M. Scheffler, Comput. Phys. Commun. 107, 187 (1997).

[13] X. Gonze, http://www.abinit.org/.

[14] H.J. Monkhorst, J.D. Pack, Phys. Rev. B 13, 5188 (1976).

[15] M. Fuchs, M. Scheffler, Comput. Phys. Commun. 119, 67 (1999).

[16] J.P. Perdew, K. Burke, M. Ernzerhof, Phys. Rev. Lett. 77, 3865 (1996).

[17] D.E. Gray, American Institute of Physics Handbook, American Institute of Physics, New York 1972.

[18] D.C. Wallace, Thermodynamics of Crystals, Dover, New York 1972.

[19] D.R. Lide, Handbook of Chemistry and Physics, CRC press, New York 1998.

[20] http://www.physics.ohio-state.edu/ wilkins/group/phases/index.html.

[21] A. Kiejna, http://www.za.ifd.uni.wroc.pl/html/kiejna.html.

[22] A. Oganov, http://olivine.ethz.ch/artem. 